\title{
WBC Associates with Readmission Following Cystectomy
}

\author{
Andrew G. McIntosh ${ }^{\mathrm{a}}$, Tianyu Li ${ }^{\mathrm{b}}$, Timothy Ito ${ }^{\mathrm{b}}$, Jason Mannion $^{\mathrm{a}}$, Mark Dziemianowicz ${ }^{\mathrm{a}}$, \\ Nikhil Waingankar ${ }^{\mathrm{b}}$, Mohammed Haseebuddin ${ }^{\mathrm{b}}$, David Y.T. Chen ${ }^{\mathrm{b}}$, Richard E. Greenberg ${ }^{\mathrm{b}}$, \\ Rosalia Viterbo $^{\mathrm{b}}$, Alexander Kutikov ${ }^{\mathrm{b}}$, Robert G. Uzzo ${ }^{\mathrm{b}, \mathrm{c}}$, Marc C. Smaldone ${ }^{\mathrm{b}}$ \\ and Philip H. Abbosh ${ }^{\mathrm{b}, \mathrm{c}, *}$ \\ ${ }^{a}$ Temple University Health System, Philadelphia, PA, USA \\ ${ }^{\mathrm{b}}$ Fox Chase Cancer Center, Philadelphia, PA, USA \\ ${ }^{\mathrm{c}}$ Albert Einstein Medical Center, Philadelphia, PA, USA
}

\begin{abstract}
.
Background: Radical cystectomy is associated with perioperative complication rates exceeding $50 \%$ in some series. Readmission rates are increasingly used as a surgical quality metric. White blood cell count is a crude surrogate for physiologic processes which may reflect postoperative complications leading to readmission.

Objective: We assessed the association between final white blood cell count at discharge and risk of readmission following radical cystectomy.

Methods: Records on 477 patients undergoing radical cystectomy from 2006-2013 were reviewed. Final white blood cell count was defined as the last documented value during index admission. Univariate analysis was performed using Fisher's exact, Wilcoxon rank sum test, and Spearman's coefficient tests where appropriate. Multivariable logistic regression models were used to test the associations between final white blood cell count and readmission.

Results: $34 \%$ of patients were readmitted within 90 days of surgery. Amongst this cohort, a cutoff final white blood cell count of $9000 / \mathrm{mm}^{3}$ was identified, with a significantly higher proportion of patients with values $>9000 / \mathrm{mm}^{3}$ experiencing readmission than those with values $\leq 9000 / \mathrm{mm}^{3}$ ( $42 \%$ vs $\left.28 \%, p=0.004\right)$. Other perioperative variables associated with an increased readmission rate included initial hospital length of stay $\leq 10$ days, and receipt of a continent diversion. Following adjustment, final white blood cell count $>9000 / \mathrm{mm}^{3}$ was associated with increased risk of readmission (OR $2.09,95 \%$ CI $1.23-3.53, p=0.006)$.

Conclusions: Final white blood cell count is associated with hospital readmission following radical cystectomy. This metric may provide important guidance in discharge algorithms.
\end{abstract}

Keywords: Urinary bladder neoplasms, cystectomy, patient readmission, leukocyte count

\section{ABBREVIATION}

${ }^{*}$ Correspondence to: Philip H. Abbosh, MD-PhD, Fox Chase Cancer Center, 333 Cottman Ave, Philadelphia, PA 19111, USA. Tel.: +1 215728 7278; Fax: +1 215214 1734; E-mail: Philip.Abbosh@fccc.edu.
$\mathrm{RC}$
Radical Cystectomy
BCG
Bacillus Calmette-Guérin
WBC 


$\begin{array}{ll}\text { FWBC } & \text { Final White Blood Cell Count } \\ \text { UVA } & \text { Univariate Analysis } \\ \text { MVA } & \text { Multivariate Analysis } \\ \text { LOS } & \text { Length of Stay } \\ \text { BMI } & \text { Body-Mass Index } \\ \text { ASA } & \text { American Society of Anesthesiologists } \\ \text { RT } & \text { Radiation Therapy }\end{array}$

\section{INTRODUCTION}

Radical cystectomy ( $\mathrm{RC}$ ) is the primary therapy for loco-regional control of muscle-invasive or BCGrefractory bladder cancer [1]. $\mathrm{RC}$ is associated with high rates of perioperative morbidity and mortality, with 90 -day complication rates ranging between $31-64 \%$ [2-8].

Complication rates after $\mathrm{RC}$ are widely reported and there is significant literature devoted to understanding the causes of readmission following RC. Early readmission rates range from 20-26\% [9-13], respectively, and complication rate is probably associated with hospital readmission $[10,13]$. Hospital readmission rates are increasingly being tied to healthcare economics and surgical quality metrics [14], highlighting the value of identifying risk factors for readmission.

White blood cell count (WBC) is a crude surrogate of inflammatory and other physiologic processes $[15,16]$, which may be associated with various causes of postoperative complications. As post discharge complications often involve inflammatory processes resulting in readmission to the hospital [9] and infectious causes have been found to account for $52 \%$ of non-modifiable readmissions [11], it may be of value to determine if a marker of inflammation during the index hospital stay can predict readmission. Our objective was to assess the association between final recorded white blood cell count (fWBC) prior to index hospitalization discharge and readmission in patients undergoing RC.

\section{MATERIALS AND METHODS}

\section{Study population}

Following exempt Institutional Review Board Approval, our institutional prospectively maintained bladder cancer database was queried for all cases of RC performed from 2006-2013 (Fig. 1). Our data reflects that of five fellowship trained bladder surgeons (one additional surgeon pre-dates formal fellowship training programs) of variable clinical volume and experience at a high-volume NCCNdesignated comprehensive cancer center. Fifty-three patients have follow-up $<90$ days, 26 of whom died within 90 days. Patients with missing data or inadequate follow up were excluded from analyses where necessary. Patients who died during their index hospitalization were also excluded from analyses.

\section{Co-Variates}

54 pre- and postoperative clinicopathologic variables were retrospectively obtained from computerized health records. These include patient demographics, pre- and postoperative laboratory values, oncologic status including pathologic staging, neoadjuvant chemotherapies, and indications for cystectomy, preoperative comorbidities, ECOG status, intraoperative factors including blood loss, and postoperative morbidity and mortality data. These variables are listed in Supplementary Table 1.

\section{Exposure and outcomes}

fWBC was defined as the last documented WBC during the index hospital admission. Admissions to the hospital within 90 days of RC were defined as hospital readmissions.

\section{Statistical analyses}

SAS 9.3 was utilized for statistical programming and analysis. Significant cutoff values for continuous variables were identified using the recursive partitioning method. When examining fWBC amongst this cohort, a cutoff value of $9.4 \times 10^{3} / \mathrm{mm}^{3}$ was identified (rounded off to $9.0 \times 10^{3} / \mathrm{mm}^{3}$ ) using threshold analysis with readmission used as the primary endpoint. Further, we divided the cohort into a discovery and validation set and repeated the classification process to identify at optimized cutoff point for fWBC. Univariate analysis (UVA) was performed using Fisher's exact, Wilcoxon rank sum test, and Spearman's coefficient tests where appropriate. For multivariate analysis (MVA), models were fit using logistic regression.

\section{RESULTS}

477 patients undergoing RC were identified. 352 (74\%) were male. The median age of the cohort was 


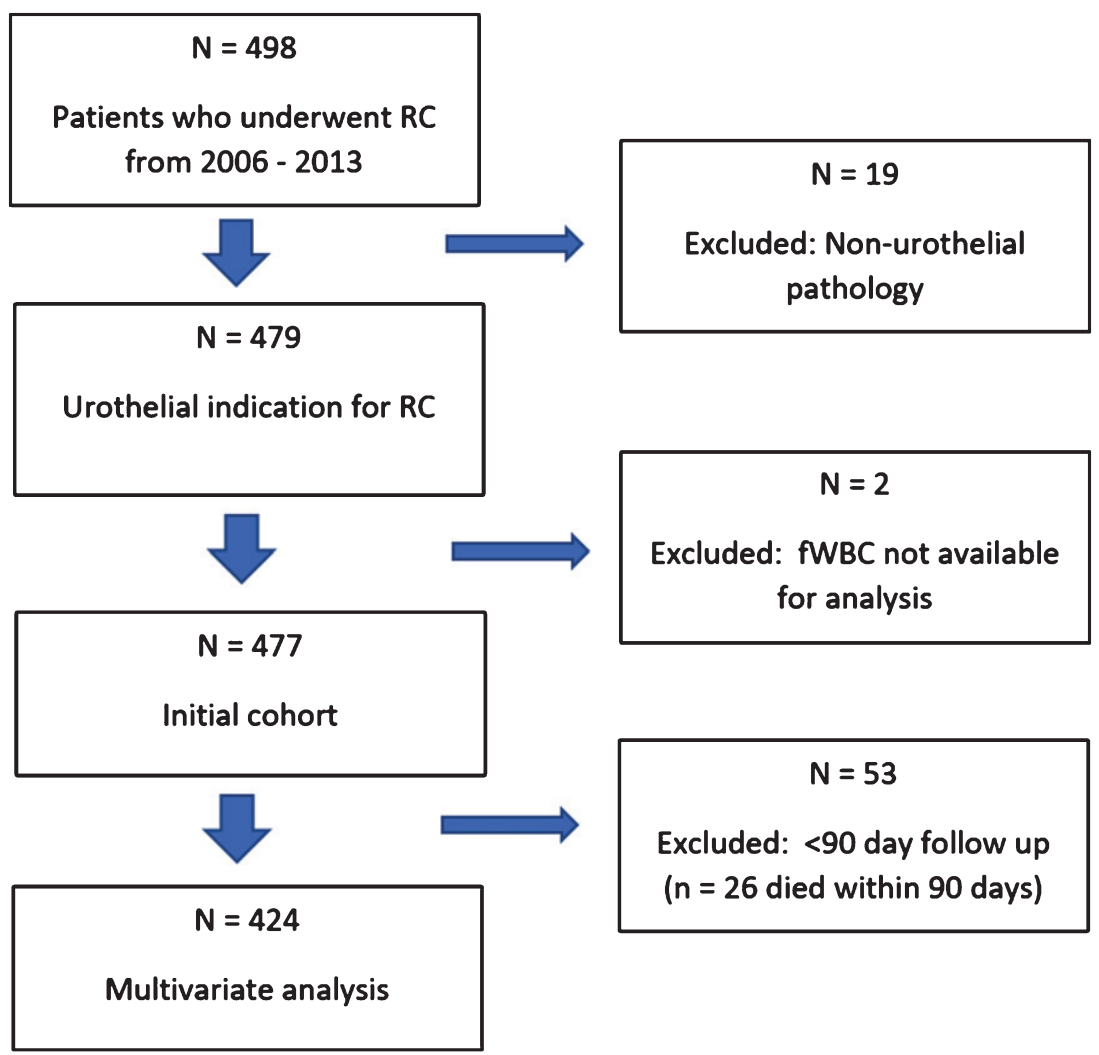

Fig. 1. Study Population.

Table 1

Baseline patient characteristics

\begin{tabular}{lcc}
\hline & $\mathrm{N}(\%)$ & Median $+/$ - Std. dev. \\
\hline Patients & 477 & $69.77+/-10.02$ \\
Age (yrs) & & \\
Male & $352(74)$ & \\
$\quad$ Male & $124(26)$ & \\
Female & & \\
Race & $422(90.2)$ & \\
$\quad$ White & $36(7.7)$ & \\
$\quad$ African-American & $10(2.1)$ & \\
Other & 454 & $26.9+/-5.9$ \\
BMI (kg/m $\left.{ }^{2}\right)$ & 469 & $12.5+/-1.86$ \\
Hgb (g/dL) & 466 & $1.03+/-0.53$ \\
Creatinine (mg/dL) & 452 & $3.95+/-0.62$ \\
Albumin (g/dL) & 477 & \\
ASA & $16(3.3)$ & \\
$\quad 1$ & $296(62.1)$ & \\
2 & $163(34.2)$ & \\
3 & $2(0.4)$ & \\
4 & $53(11.1)$ & \\
h/o XRT* & 488 & $600+/-983.39$ \\
EBL (cc) &
\end{tabular}

*XRT for prostate or colorectal cancer.

69.5 years. 366 patients had fWBC measured on their final day of admission and the other 111 patients had fWBC measured at least one day prior. Peri-operative demographics and outcomes are listed in Tables 1 and 2. Patients who suffered complications during the index admission were categorized by complication type (Table 3 ). A total of 86 patients (18\%) had a complication during their index hospital stay, 60 of whom qualified for multiple complication categories. As an example, a patient with a pulmonary embolism would qualify for both the DVT/PE category and pulmonary category. Infectious complications were the most common category comprising 39 patients $(8.1 \%)$, followed by 22 patients $(4.6 \%)$ and 21 patients $(4.4 \%)$ who had gastro-intestinal complications and a return to the operating room, respectively.

In total, 164 (34.4\%) of patients were readmitted within 90 days of RC and $26(5 \%)$ were deceased within 90 days of surgery. Table 4 describes the reasons for readmission with the most common etiology being infectious/inflammatory in nature in 106 patients $(22.2 \%)$. Of the 26 patients who died, $11(44 \%)$ expired due to complications related to surgery, as opposed to five (20\%) who died from progression of disease and three (12\%) who died from complications related to co-morbid conditions. Seven patients $(28 \%)$ who died within 90 days were lost 


\section{Discovery set}

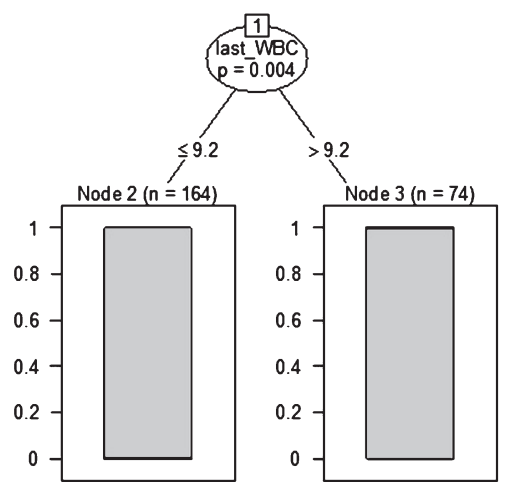

Original cohort

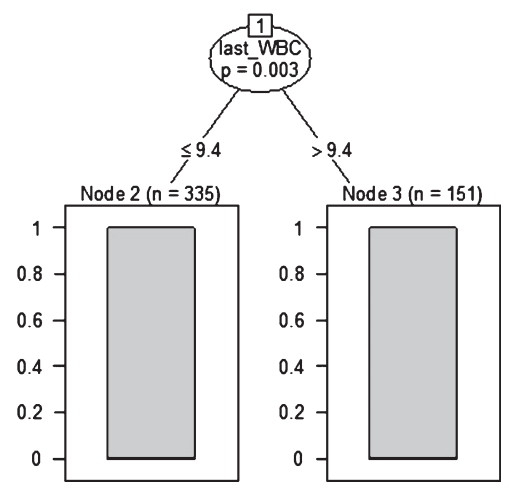

Fig. 2. Discovery set ( $50 \%$ of the cohort) vs. original result.

to follow up prior to their death. Nine patients died during their index hospitalization.

When examining fWBC amongst this cohort, a cutoff value of $9000 / \mathrm{mm}^{3}$ was identified using threshold analysis. Amongst the cohort, 37\% of patients had a fWBC of $>9000 / \mathrm{mm}^{3}$. A significantly higher proportion of patients with $\mathrm{fWBC}>9000 / \mathrm{mm}^{3}$ experienced readmission (Table 5) than those with fWBC $\leq 9000 / \mathrm{mm} 3$ ( $42 \%$ vs $28 \%, p=0.004)$. Other perioperative variables associated with an increased readmission rate included initial hospital length of stay $\leq 10$ days ( $36 \%$ vs $26 \%, p=0.04)$, and continent diversion $(51 \%$ vs $30 \%, p<0.0001)$. Overall complication rates during the index admission were not associated with readmission $(25 \%$ vs $35 \%$, $p=0.09$ ). Infectious complications accounted for over $40 \%$ of all patients readmitted to the hospital and when combined with other inflammatory conditions such as DVT/PE, accounted for nearly two-thirds of readmissions. However, readmission for infectious/inflammatory conditions was not different among fWBC groups (9\% vs. 11.2\%, $p=0.43$ ).

When evaluating for an association between LOS and readmission for infectious/inflammatory conditions, we found that $11.6 \%$ of patients with a LOS of $\leq 10$ days were readmitted for infectious/inflammatory etiologies, compared to only $6 \%$ of those patients with LOS $>10$ days. This, however, only approached statistical significance $(p=0.058)$. We also found no difference between median fWBC for LOS $\leq 10$ days vs. $>10$ days ( 8 vs 8.6 days, $p=0.1$ ).

When evaluating comparisons between fWBC and other investigated variables, we noted patients with $\mathrm{fWBC}>9000 / \mathrm{mm}^{3}$ were more likely to have a Clavien grade 3 complication (39.9\% vs. $29.1 \%$, $p=0.02)$ and to die within 90 days of surgery $(9.6 \%$ vs. $3 \%, p=0.003)$. fWBC was not associated with significant differences between in-house complications, LOS, type of diversion (continent vs. incontinent), open vs. robotic surgery, sex, race, renal function, ASA, or radiation history.

On multivariate analysis for predictors of readmission, controlling for significant variables (Table 6), fWBC $>9000 / \mathrm{mm}^{3}$ remained an independent predictor of readmission post-RC (OR 2.09, 95\% CI $1.23-3.53, p=0.006)$. Continent diversion (OR 2.50, $95 \%$ CI $1.21-5.18, p=0.01)$ and shorter initial hospital length of stay ( $\leq 10$ days) (OR $4.03,95 \%$ CI $1.99-8.14, p=0.0001)$ also remained significant predictors of readmission.

After dividing the cohort randomly and equally into a discovery set and validation set, the classification process on the discovery set was repeated to look for an optimized cut-off point for fWBC. We obtained a value of $9.2 \times 10^{3} / \mathrm{mm}^{3}$ which was similar to our previous finding of $9.4 \times 10^{3} / \mathrm{mm}^{3}$ (Fig. 2). Using the rounded value of $9.0 \times 10^{3} / \mathrm{mm}^{3}$ to fit an MVA model using the validation data, we produced similar results to our MVA previously described in Table 6. We have included our validation set MVA in Supplementary Table 2.

\section{DISCUSSION}

We sought to determine whether fWBC was associated with hospital readmission following RC. Further, we aimed to analyze 54 clinicopathologic variables obtained from review of our bladder cancer database to identify other factors that associated with readmission following RC. We found that $\mathrm{WBC}$ above $9000 / \mathrm{mm}^{3}$ was significantly associated with risk of 
Table 2

Perioperative demographics and outcomes

\begin{tabular}{lc}
\hline & Total $(\%)$ \\
\hline WBC $\left(\times 10^{9} / \mathrm{L}\right)$ & $299(62.7)$ \\
$\quad<=9$ & $178(37.3)$ \\
$>9$ & $158(33.12)$ \\
Readmission (90 days) & $84(17.61)$ \\
Complication (in-house) & $158(33.12)$ \\
Clavien III complication & $26(5.45)$ \\
Death (90 days) & \\
Length of stay (days) & $327(68.7)$ \\
$0-10$ & $149(31.3)$ \\
$>10$ & $88(18.5)$ \\
MIS & \\
Diversion & $404(84.9)$ \\
$\quad$ Incontinent & $72(15.1)$ \\
$\quad$ Continent & $58(12.2)$ \\
Live discharge other than home & \\
(e.g. skilled nursing facility, acute rehab, etc) & \\
\hline
\end{tabular}

hospital readmission. Presence of a continent diversion andinitial hospital LOS $<10$ days also predicted readmission.

In a recent review by Richards et al. [5], the authors meticulously describe a number of pre-, post-, and intra-operative considerations that can be used to minimize the significant post-operative morbidity of RC and to appropriately risk-stratify patients. The complication profile following radical cystectomy has been associated with preoperative comorbidity status, age, and oncologic status [2, 4]. Additionally, preoperative renal insufficiency and high intraoperative intravenous fluid administration have been associated on multivariate analysis with complications of any grade while increasing intraoperative blood loss was associated with high-grade complications [17]. Furthermore, functional status, preoperative dyspnea, chronic steroid use, preoperative alcohol consumption, operative time, and surgeon level of training [18] are amongst many other [6] components of the perioperative patient profile that can be considered.

Most of the previously published cohorts focused attention on pre-operative risk factors that stratify patients into higher risk groups for post-operative complications such as increasing BMI, Charleson co-morbidity index, or ASA score [3]. However, readmission rates, or specific perioperative factors that predict readmission, are far less frequently reported [9].

The combination of a high rate of adverse events associated with significant economic burden [19, 20] and a high rate of readmission warrants closer attention, especially in the context of increasing scrutiny being leveled upon hospital readmission rates. For complex oncologic surgical procedures such as RC, identification of easy-to-measure clinical parameters associated with risk of re-hospitalization - like WBC - may help to guide targeted interventions in the outpatient setting to reduce readmission risk. This approach complements enhanced recovery after surgery protocols that have been shown to shorten hospital stay without increasing readmission rates [21].

WBC is a crude surrogate for generalized inflammatory processes in many urological and nonurological diagnoses. WBC associates with hospital readmission rates in patients with renal colic [22] and pulmonary embolism [23]. Interestingly, elevated pre-operative $\mathrm{WBC}$ has also been independently associated with increased adverse postoperative outcomes and mortality [15, 24], and with the development of post-operative atrial fibrillation [25] following coronary artery bypass grafting.

The results of our study show that fWBC is associated with risk of hospital readmission following RC. This may suggest that these patients have demonstrated a generalized increase in their acute phase response, heralding an otherwise unforeseen future infectious or otherwise inflammatory complication. This conclusion is especially valuable given that a large proportion of hospital readmissions following $\mathrm{RC}$ are a result of infectious complications.

Although we did note that nearly two-thirds of readmissions were due to infectious/inflammatory complications, our analysis did not show a specific association between infectious/inflammatory readmissions and fWBC. This may be due to the fact that active infectious/inflammatory complications that occur during the index stay are associated with other clinical signs/symptoms and would be recognized/treated prior to discharge, whereas sterile inflammatory processes may be more insidious. Patients with clinical symptoms (e.g. fever) are less likely to be discharged with a persistently elevated WBC. However, subtle harbingers of an elevated WBC (e.g. pelvic lymphocele) that may lead to an eventual infectious process or trigger readmission could be clinically silent at the time of initial discharge.

Our analysis also demonstrated that an initial hospital $\operatorname{LOS} \leq 10$ days was associated with risk of readmission. This suggests that those patients who are ultimately readmitted may have benefitted from a more prolonged index hospitalization. While prolonged hospital stays have adverse costs, avoidance 
Table 3

In-House Complications

\begin{tabular}{lcc}
\hline Category & $\begin{array}{c}\text { Number of patients } \\
\text { qualifying for category }\end{array}$ & $\%$ of cohort $(n=477)$ \\
\hline Infectious & 39 & 8.1 \\
Gastro-intestinal & 22 & 4.6 \\
Return to OR & 21 & 4.4 \\
Cardiovascular & 15 & 3.1 \\
Pulmonary & 11 & 2.3 \\
DVT/PE & 10 & 2.1 \\
Bleeding & 8 & 1.7 \\
Intra-operative complication & 7 & 1.5 \\
Death & 6 & 1.3 \\
Renal/electrolytes & 4 & 0.8 \\
Other & 2 & 0.4 \\
Disease progression & 1 & 0.2 \\
Patients qualifying for multiple categories & 60 & 12.6 \\
Total complications & 146 & - \\
Total number of patients & 86 & 18.0 \\
\hline
\end{tabular}

Table 4

Readmission within 90 days

\begin{tabular}{lcc}
\hline Category & Number of patients & \% of cohort in category $(N=477)$ \\
\hline Infectious/inflammatory & 106 & 22.2 \\
Renal/electrolytes/FTT & 63 & 13.0 \\
Other (Pain, social issues, disease progression, & 12 & 2.5 \\
$\quad$ complication NOS) & & \\
CV/Pulmonary (non-infectious) & 8 & 1.7 \\
Patients in multiple readmission categories & 25 & 5.2 \\
Total readmissions & 164 & 34.3 \\
\hline
\end{tabular}

of immediate hospital readmission offsets these costs and would avoid financial penalties for hospital readmission.

Recognizing the limitations of fWBC as a nonspecific biomarker for readmission, and acknowledging that there are a multitude of etiologies for post-operative inflammatory processes, fWBC may be best used in conjunction with other clinical variables to guide decision-making. It is not uncommon for WBC to fluctuate in the post-operative period without a clear explanation. Our results suggest that these fluctuations might be considered when decisions regarding disposition are being made. For example, if a patient has an inflammatory or infectious complication, fWBC may be useful in conjunction with other parameters - as a measure of suitability for discharge. Further, fWBC could be used to risk-stratify patients at the time of discharge and inform tailored interventions in the outpatient setting such as home fluid resuscitation or identify patients who would benefit from more immediate follow up evaluation. This argument is supported by the fact that readmissions for infectious/inflammatory conditions were more likely when LOS was shorter. Although this only approached sta- tistical significance, we believe that this finding is clinically significant and when combined with our other findings makes the case that some patients may benefit from a more protracted inpatient stay if an infectious/inflammatory condition is suspected. We further noted that $\mathrm{fWBC}>9000 / \mathrm{mm}^{3}$ predicted the presence of a Clavien grade 3 or greater complication and death within 90 days of surgery, further highlighting the potential significance of fWBC.

Our findings are limited primarily by the retrospective institutional study design.

One other limitation of our study is that we did not capture all outside hospital readmissions. However, the institutional practice is that every effort is made to transfer patients back to Fox Chase Cancer Center if they are admitted to a non-index hospital. Further, if the readmission was brought to the attention of the staff, it was documented in the prospective database.

It is intuitive that the absence of leukocytosis alone should not guide the disposition of patients and it is also noteworthy that a WBC of $9000 / \mathrm{mm}^{3}$ is not considered to be abnormal. However, in the appropriate clinical context it can be used as an adjunct to other objective and subjective data to adjudicate 
Table 5

Univariate Analysis for predictors of readmission

\begin{tabular}{|c|c|c|c|c|}
\hline & \multicolumn{4}{|c|}{ Readmission } \\
\hline & $\mathrm{n}$ & Yes $(\%)$ & No $(\%)$ & $p$-value \\
\hline WBC & & & & $P=0.004$ \\
\hline$<=9 / \mathrm{mm} 3$ & 299 & $84(28)$ & $215(72)$ & \\
\hline$>9 / \mathrm{mm} 3$ & 178 & $74(42)$ & $104(58)$ & \\
\hline In-house complication & & & & $P=0.09$ \\
\hline Yes & 84 & $21(25)$ & $63(75)$ & \\
\hline No & 393 & $137(35)$ & $256(65)$ & \\
\hline LOS & & & & $P=0.04$ \\
\hline $0-10 \mathrm{~d}$ & 327 & $118(36)$ & 209 (64) & \\
\hline$>10 \mathrm{~d}$ & 149 & $39(26)$ & $110(74)$ & \\
\hline Surgery type & & & & $P=0.71$ \\
\hline Open & 389 & $127(33)$ & $262(67)$ & \\
\hline MIS & 88 & $31(35)$ & $57(64)$ & \\
\hline Diversion type & & & & $P<0.0001$ \\
\hline Incontinent & 404 & $120(30)$ & $284(70)$ & \\
\hline Continent & 72 & $37(51)$ & $35(49)$ & \\
\hline Clavien III or higher & & & & $P<0.0001$ \\
\hline Yes & 158 & $98(62)$ & $60(38)$ & \\
\hline No & 319 & $60(19)$ & $259(81)$ & \\
\hline Death $<90 \mathrm{~d}$ & & & & $P=0.08$ \\
\hline Yes & 26 & $13(50)$ & $13(50)$ & \\
\hline No & 451 & $145(32)$ & $306(68)$ & \\
\hline Sex & & & & $P=0.12$ \\
\hline Male & 352 & $124(35)$ & $228(65)$ & \\
\hline Female & 124 & $34(27)$ & $90(73)$ & \\
\hline Race & & & & $P=0.35$ \\
\hline White & 422 & $137(32)$ & $285(68)$ & \\
\hline AA & 36 & $16(44)$ & $20(56)$ & \\
\hline Other & 10 & $3(30)$ & $7(70)$ & \\
\hline Disposition at discharge & & & & $p=0.04$ \\
\hline Nursing home & 58 & $12(21)$ & $46(79)$ & \\
\hline Other & 419 & $146(35)$ & $273(65)$ & \\
\hline History of pelvic XRT & & & & $P=0.89$ \\
\hline Yes & 53 & $18(34)$ & $35(66)$ & \\
\hline No & 424 & $140(33)$ & $284(67)$ & \\
\hline ASA & & & & $P=0.21$ \\
\hline 1 & 16 & $3(19)$ & $13(81)$ & \\
\hline 2 & 296 & $92(31)$ & $204(69)$ & \\
\hline 3 & 163 & $62(38)$ & $101(62)$ & \\
\hline 4 & 2 & $1(50)$ & $1(50)$ & \\
\hline
\end{tabular}

*Indication for majority of prior radiation was prostate cancer.

Table 6

Multivariate analysis for predictors of readmission

\begin{tabular}{|c|c|c|c|c|}
\hline Effect & Hazard ratio & 95\% Confidence Limits & & $P$ value \\
\hline WBC trend (stable/decreasing vs. rising) & 1.11 & 0.66 & 1.89 & 0.70 \\
\hline WBC group $\left(>9 \mathrm{k} / \mathrm{mm}^{3}\right.$ vs. $\left.\leq 9 \mathrm{k} / \mathrm{mm}^{3}\right)$ & 2.09 & 1.23 & 3.53 & 0.006 \\
\hline Diversion (continent vs. incontinent) & 2.50 & 1.21 & 5.18 & 0.01 \\
\hline Dicharge disposition (other vs. nursing home) & 1.44 & 0.61 & 3.41 & 0.41 \\
\hline LOS $(0-10 \mathrm{~d}$ vs. $>10 \mathrm{~d})$ & 4.03 & 1.99 & 8.136 & 0.0001 \\
\hline
\end{tabular}

discharge strategy and follow-up. The association of a shortened index length of stay with risk for readmission further asserts that patients may benefit by being appropriately triaged into higher-risk groups and subsequently having their index stay prolonged where appropriate. By using objective risk predictors like fWBC, the economic burden of frequent hospital readmission after $\mathrm{RC}$ on hospital resources may be diminished.

\section{CONCLUSIONS}

In our cohort of patients undergoing RC, fWBC was associated with increased hospital readmission 
rates. Use of this readily available clinical metric, combined with an overall clinical assessment, may afford opportunities to tailor hospital discharge strategies and allow for a more informed triage of $\mathrm{RC}$ patients into appropriate outpatient follow-up algorithms.

\section{CONFLICT OF INTEREST}

The authors have no conflict of interest to report.

\section{FUNDING}

Philip Abbosh: Urology Care Foundation, Kidney Cancer Association.

\section{SUPPLEMENTARY MATERIAL}

The supplementary material is available in the electronic version of this article: http://dx.doi.org/ 10.3233/BLC-160088.

\section{REFERENCES}

[1] Gakis G, Efstathiou J, Lerner SP, Cookson MS, Keegan KA, Guru KA, Shipley WU, Heidenreich A, Schoenberg MP, Sagaloswky AI, Soloway MS, Stenzl A. ICUDEAU International Consultation on Bladder Cancer 2012: Radical Cystectomy and Bladder Preservation for MuscleInvasive Urothelial Carcinoma of the Bladder. Eur Urol 2013;63(1):45-57.

[2] Hautmann RE, de Petriconi RC, Volkmer BG. Lessons learned from 1,000 neobladders: The 90-day complication rate. J Urol 2010;184(3):990-4; quiz 1235.

[3] Roghmann F, Trinh QD, Braun K, von Bodman C, Brock M, Noldus J, Palisaar J. Standardized assessment of complications in a contemporary series of European patients undergoing radical cystectomy. International Journal of Urology: Official Journal of the Japanese Urological Association 2014;21(2):143-9.

[4] Gandaglia G, Varda B, Sood A, Pucheril D, Konijeti R, Sammon JD, Sukumar S, Menon M, Sun M, Chang SL, Montorsi F, Kibel AS, Trinh QD. Short-term perioperative outcomes of patients treated with radical cystectomy for bladder cancer included in the National Surgical Quality Improvement Program (NSQIP) database. Canadian Urological Association Journal=Journal de l'Association Des Urologues Du Canada 2014;8(9-10):E681-E7.

[5] Richards KA, Steinberg GD. Perioperative outcomes in radical cystectomy: How to reduce morbidity? Curr Opin Urol 2013;23(5):456-65.

[6] Lavallee LT, Schramm D, Witiuk K, Mallick R, Fergusson D, Morash C, Cagiannos I, Breau RH. Peri-operative morbidity associated with radical cystectomy in a multicenter database of community and academic hospitals. PLoS One one 2014;9(10):e111281.
[7] Hautmann RE, Hautmann SH, Hautmann O. Complications associated with urinary diversion. Nat Rev Urol 2011;8(12):667-77.

[8] Novara G, De Marco V, Aragona M, Boscolo-Berto R, Cavalleri S, Artibani W, Ficarra V. Complications and mortality after radical cystectomy for bladder transitional cell cancer. J Urol 2009;182(3):914-21.

[9] Stimson CJ, Chang SS, Barocas DA, Humphrey JE, Patel SG, Clark PE, Smith JA, Jr., Cookson MS. Early and late perioperative outcomes following radical cystectomy: 90-day readmissions, morbidity and mortality in a contemporary series. J Urol 2010;184(4):1296-300.

[10] Hu M, Jacobs BL, Montgomery JS, He C, Ye J, Zhang Y, Brathwaite J, Morgan TM, Hafez KS, Weizer AZ, Gilbert SM, Lee CT, Lavieri MS, Helm JE, Hollenbeck BK, Skolarus TA. Sharpening the focus on causes and timing of readmission after radical cystectomy for bladder cancer. Cancer 2014;120(9):1409-16.

[11] James AC, Izard JP, Holt SK, Calvert JK, Wright JL, Porter MP, Gore JL. Root Causes and Modifiability of 30-Day Hospital Readmissions after Radical Cystectomy for Bladder Cancer. J Urol 2015;195(4):894-9.

[12] Nayak JG, Gore JL, Holt SK, Wright JL, Mossanen M, Dash A. Patient-centered risk stratification of disposition outcomes following radical cystectomy. Urol Oncol 2015;35(5):17-23.

[13] Skolarus TA, Jacobs BL, Schroeck FR, He C, Helfand AM, Helm J, Hu M, Lavieri M, Hollenbeck BK. Understanding hospital readmission intensity after radical cystectomy. J Urol 2015;193(5):1500-6.

[14] Greenberg L. Reducing Hospital Readmissions After Surgery: A Brief for Preferred Provider Organizations 2012. Available from: http://www.aappo.org/Portals/0/ Documents/GRANTS/Issue\%20Brief.pdf.

[15] Dacey LJ, DeSimone J, Braxton JH, Leavitt BJ, Lahey SJ, Klemperer JD, Westbrook BM, Olmstead EM, O'Connor GT. Preoperative white blood cell count and mortality and morbidity after coronary artery bypass grafting. The Annals of Thoracic Surgery 2003;76(3):760-4.

[16] Tang C, Gomez DR, Wang H, Levy LB, Zhuang Y, Xu T, Nguyen Q, Komaki R, Liao Z. Association between white blood cell count following radiation therapy with radiation pneumonitis in non-small cell lung cancer. International Journal of Radiation Oncology, Biology, Physics 2014;88(2):319-25

[17] Kauffman EC, Ng CK, Lee MM, Otto BJ, Portnoff A, Wang GJ, Scherr DS. Critical analysis of complications after robotic-assisted radical cystectomy with identification of preoperative and operative risk factors. BJU International 2010;105(4):520-7.

[18] Hollenbeck BK, Miller DC, Taub D, Dunn RL, Khuri SF, Henderson WG, Montie JE, Underwood W, 3rd, Wei JT. Identifying risk factors for potentially avoidable complications following radical cystectomy. J Urol 2005;174(4 Pt 1):1231-7; discussion 7.

[19] Kim SP, Shah ND, Karnes RJ, Weight CJ, Frank I, Moriarty JP, Han LC, Borah B, Tollefson MK, Boorjian SA. The implications of hospital acquired adverse events on mortality, length of stay and costs for patients undergoing radical cystectomy for bladder cancer. J Urol 2012;187(6): 2011-7.

[20] Konety BR, Allareddy V. Influence of post-cystectomy complications on cost and subsequent outcome. J Urol 2007;177(1):280-7; discussion 7. 
[21] Daneshmand S, Ahmadi H, Schuckman AK, Mitra AP, Cai J, Miranda G, Djaladat H. Enhanced recovery protocol after radical cystectomy for bladder cancer. J Urol 2014;192(1):50-5.

[22] Sfoungaristos S, Hidas G, Gofrit ON, Rosenberg S, Yutkin V, Landau EH, Pode D, Duvdevani M. A novel model to predict the risk of readmission in patients with renal colic. Journal of Endourology / Endourological Society 2014;28(8):1011-5.

[23] Venetz C, Labarere J, Jimenez D, Aujesky D. White blood cell count and mortality in patients with acute pulmonary embolism. Am J Hematol 2013;88(8):677-81.
[24] Newall N, Grayson AD, Oo AY, Palmer ND, Dihmis WC, Rashid A, Stables RH. Preoperative white blood cell count is independently associated with higher perioperative cardiac enzyme release and increased 1-year mortality after coronary artery bypass grafting. The Annals of Thoracic Surgery 2006;81(2):583-9.

[25] Fontes ML, Amar D, Kulak A, Koval K, Zhang H, Shi W, Thaler H. Increased preoperative white blood cell count predicts postoperative atrial fibrillation after coronary artery bypass surgery. Journal of Cardiothoracic and Vascular Anesthesia 2009;23(4):484-7. 